\title{
Substructure in the Galactic Halo
}

\author{
Steven R. Majewski ${ }^{1,2}$ \\ ${ }^{1}$ Department of Astronomy, University of Virginia, Charlottesville, VA 22903-0818, USA \\ ${ }^{2}$ E-mail: srm4n@didjeridu.astro.virginia.edu
}

Received 2003 December 23, accepted 2004 March 24

\begin{abstract}
Giant stars make particularly useful tracer stars for halo substructure because they are very bright and very common. I discuss several projects that use giant-star tracers to search the Galactic halo for tidal debris from known Galactic satellites, including that from the Sagittarius dwarf galaxy, and to search for tidal debris features from former, now destroyed satellites. Several cross-sections of the halo reveal it to be networked with extended, coherent substructures, indicating that it is likely to be predominantly made up of accreted satellites.
\end{abstract}

Keywords: Galaxy: kinematics and dynamics — Galaxy: structure — Galaxy: halo

\section{Giant Stars as Tracers of Halo Substructure}

Current cold dark matter (CDM) based structure formation models suggest that Milky Way-like galaxies should presently contain substantial halo substructure as a result of the accretion of numerous subhalos over their lifetimes (e.g., Navarro, Frenk, \& White 1997). This notion that halos like that of the Milky Way (MW) contain the residue of formerly independently evolving, proto-Galactic 'fragments' was anticipated, using stellar populations arguments, by Searle \& Zinn (1978). The recent discovery of coherent tidal streams in both the Milky Way (MW) and M31 not only provides prima facie support for hierarchical halo formation models, but allows detailed study of what is clearly an ongoing process of halo-building at zero redshift.

The degree of halo substructure in today's MW halo is a signature of the cumulative destruction history of satellite systems and bears on a number of issues: How important has accretion been over the course of MW evolution? How has the accretion rate evolved? Are presently known MW satellites being tidally disrupted? How much of the Galactic halo was formed by this process rather than by initial proto-Galactic collapse (e.g., Eggen, Lynden-Bell, \& Sandage 1962)?

Only a dozen MW satellite galaxies are now visible, whereas CDM models predict there should be hundreds. Are the 'missing' subhalos simply dark (Kauffmann, White, \& Guiderdoni 1993; Klypin et al. 1999; Moore et al. 1999), have they been more efficiently destroyed than predicted, or are the CDM models simply wrong in predicting so many subhalos agglomerated to form a MW-like galaxy?

Here I summarise results from several ongoing surveys of halo substructure aimed at answering such questions. This work, in collaboration with numerous colleagues and students at the University of Virginia and elsewhere, reveals a MW halo networked with a rich tapestry of tidal debris streams. Our strategy has been to explore substructure with $\mathrm{K}$ - and M-giant tracers because these are intrinsically bright and allow us to explore the Galaxy to large distances with even rather modest-sized telescopes. Moreover, K-giants are a stellar species found in stellar populations of every age and metallicity, so that all stellar substructure is potentially traceable by them. M-giants, on the other hand, are suitable tracers for only $[\mathrm{Fe} / \mathrm{H}] \gtrsim-1$ populations.

Tidal disruption of luminous satellites in the outer Galaxy produces long-lived, dynamically cold tidal streams that are useful probes of the size, shape, and lumpiness of the Galactic potential. Thus, systematic surveys of substructure provide new insights into both the luminous and dark Milky Way. In the interests of space I focus here simply on the appearance of substructure in the halo; application of tidal streams to determining the shape and mass of the Milky Way as well as those of the parent satellites themselves is not extensively addressed herein (but see, for example, Majewski 2004; Majewski et al. 2003a).

\section{Targeted Search for Present MW Substructure Contributors}

An obvious place to look for luminous halo substructure is in the environment of known Milky Way satellites. A challenge to determining whether these systems are in a state of tidal disruption is the extremely low surface brightness $\left(\Sigma_{V}>31 \mathrm{mag} \operatorname{arcsec}^{-2}\right)$ of their outlying regions, where tidal stripping occurs. The high density of foreground disk stars swamps any even more tenuous extratidal debris from these systems.

Our strategy for improving the signal-to-noise $(S / N)$ of such tenuous features is to identify the foreground star 'noise' and remove it. Because the foreground is typically Galactic-disk dwarf stars, we rely on two photometric 
methods that can discriminate them from the giant stars in the distant dwarf spheriodals (dSphs) and their environs:

1. The Washington $M, T_{2}+D D O 51$ filter technique (Majewski et al. 2000a), which relies on the surface gravity sensitivity of the $\mathrm{Mgb}+\mathrm{MgH}$ spectral features near $5150 \AA$ in late G- and K-type stars (the feature is secondarily sensitive to metallicity).

2. The near infrared $J H K_{\mathrm{s}}$ two-colour diagram, which separates M-dwarf and M-giant stars due to gravityinduced opacity variations primarily occurring in the $H$-band (e.g., Bessell \& Brett 1988).

Both techniques allow identification of 'needle-in-thehaystack' distant giant stars in the Galactic halo, and, when the giant star samples are further constrained to conform to the Red Giant Branch sequence in the colour-magnitude diagram of a particular satellite system, a very high confidence catalogue of satellite-associated giant stars can be generated. My group at Virginia and collaborators elsewhere have used these methods to map a number of MW and M31 satellites to equivalent surface brightnesses of $\Sigma_{V}=33$ mag $\operatorname{arcsec}^{-2}$ or fainter. The giant star catalogues for these satellites also provide relatively pure target samples for spectroscopic studies of the dynamics of the dSph systems to large radii.

The giant star density distributions of each MW dSph or globular cluster satellite we have studied (so far including the Ursa Minor, Carina, Leo II, Leo I, Sculptor, and Sagittarius dSphs, and the globulars NGC 288 and Palomar 13) exhibit radial profiles (see examples in Majewski 2003) that can be described by King (1962) profiles to nearly the King limiting radius, but then have a break to a less steep, power-law decline, extending to the limit of our ability to trace the system. These radial profiles resemble those of model disrupting satellites in MW-like potentials (e.g., Johnston, Sigurdsson, \& Hernquist 1999; Mayer et al. 2002; Johnston, Choi, \& Guhathakurta 2002), where the breaks generally correspond to a transition to recently lost, tidal tail material from the dSph. Such 'break populations' were seen or hinted at for many of the same systems we have studied in the earlier photographic analysis by Irwin \& Hatzidimitriou (1995).

Nevertheless, there remains some controversy over these results, particularly with regard to the variously studied Carina dSph, where our own work (Majewski et al. 2000b) indicates a strong break population (extending to several King limiting radii), consistent with both the earlier CCD work by Kuhn, Smith, \& Hawley (1996) as well as Irwin \& Hatzidimitriou (1995). However, the reality of our Carina break population has been questioned by Morrison et al. (2001). Unfortunately, to argue away the excess population in our data, these authors had to make several incorrect assumptions about our survey (including a substantial inflation of our typical photometric errors) as well as take advantage of a mathematical error (normalising our extratidal density to the area of our full survey, rather than just the extratidal area). More recently, Walcher et al. (2003), while claiming to find possible extratidal debris around the Sculptor $\mathrm{dSph}$, could not confirm the Carina break population. A possible reason is that their maps are created from starcounts in only one photometric band; this provides no means to photometrically eliminate any of the substantial foreground contamination, which limits the density $S / N$ in the faint outer regions of this low latitude $\mathrm{dSph}$.

In the meantime, (1) we have managed to verify spectroscopically (with Hydra fiber data from the 4-m CTIO on a subsample of our stars) that our 'success rate' for identifying Carina giant stars (based on having the correct radial velocities for Carina stars) is $>90 \%$. Presently we have nine verified Carina stars lying between 1.0 and 1.5 King limiting radii of the satellite. (2) We have obtained entirely new CTIO 4-m MOSAIC imaging of Carina which confirms our previous radial profile result (Muñoz et al., in preparation). In addition, (3) in collaboration with P. Guhathakurta we have obtain Keck spectra of 31 And I and 14 And I dSph giants identified with the same methodology as the other dSphs (Ostheimer et al., in preparation) and found 43 of 45 of these stars to be correctly identified as belonging to the And dSphs (the remaining two stars are M31 halo giants!); (4) we have obtained Keck spectra that verify $100 \%$ correct identifications among 14 Leo I giants found with our method, including eight 'extratidal' stars (Sohn et al., in preparation); while (5) Keck spectroscopy of Ursa Minor 'extratidal' stars (in collaboration with J. Kuhn) has verified fourteen of them stretching to 2.5 times the King limiting radius of that system.

Thus, the reality of associated stars lying outside the nominal King limiting radii of at least some MW satellites is without doubt. But do they really represent tidally stripped, unbound stars or are they some kind of bound 'halo' around each dSph? One clue comes from the example of the Sagittarius (Sgr) dSph, which shows the same radial profile shape as the other dSphs (Majewski et al. 2003b, hereafter MSWO). In the case of Sgr the break population is obviously from the presence of tidal debris. Either a second mechanism needs to be invoked to explain the break populations in other dSph systems, or we can look to the more straightforward explanation that all dSph breaks represent new tidal debris substructure being contributed to the MW. It may seem surprising that a MW dSph as distant $(259 \mathrm{kpc})$ as Leo I could be experiencing tidally induced disruption, but it is for this particular dSph, in fact, that we (Sohn et al., in preparation) have among the strongest indication beside Sgr that the break population takes the form of tidal 'arms' pulled out along the major axis. A 'double-break' density profile of these arms is consistently explained by mass loss during two perigalactic passages, which is the largest number of inner Galactic visits possible in a Hubble time for this high velocity satellite on what can only be a very elongated orbit. Interestingly, our calculated timings for these visits matches well the ages of two starbursts in Leo I. 


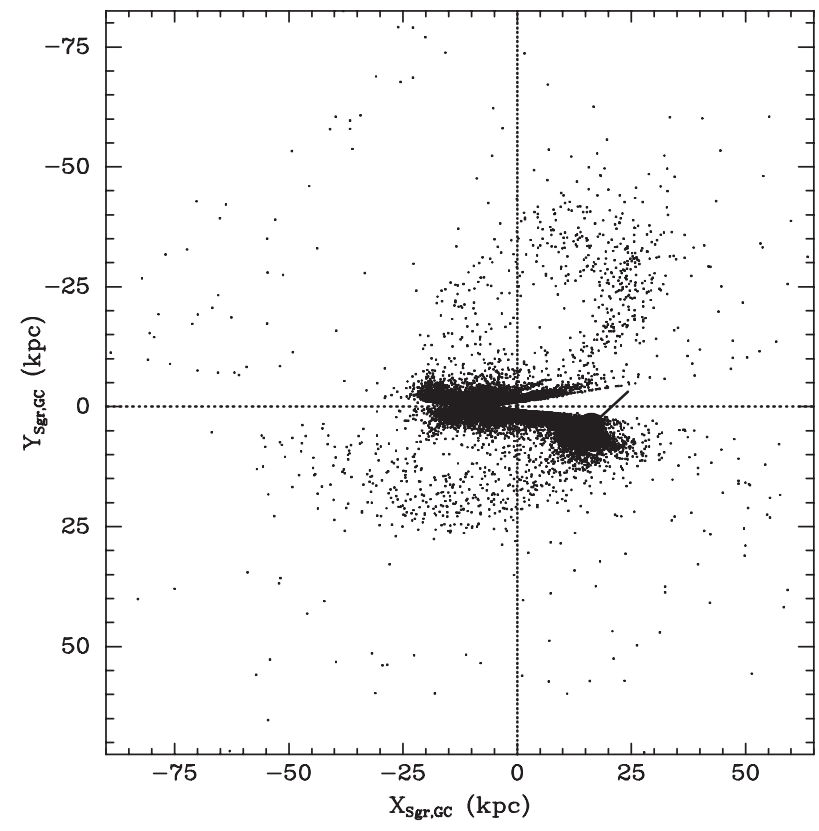

Figure 1 The distribution of M-giant stars lying within $7 \mathrm{kpc}$ of the nominal Sgr orbital plane, from MSWO. The figure is a projection onto this orbital plane, which is tipped by about $77^{\circ}$ with respect to the Galactic plane. In the figure, the Galactic disk lies along $Y_{\mathrm{Sgr}, \mathrm{GC}}=0$, and stars near this plane have been removed due to an $E(B-V)=0.555$ reddening limit, which has created the density gap to the right. The Galactic Centre is at the origin and the Sgr core is to the right. The line extending from the Sgr core indicates its direction of motion. The Sun lies at approximately $(X, Y)_{\mathrm{Sgr}, \mathrm{GC}}=(-8.5,0)$ (see Figure 3).

\section{The Sagittarius Tidal Tail System}

The Sgr system is the archetype of a dwarf galaxy merger and one that can be explored in exquisite detail. Moreover, the extensive Sgr tidal tail system gives sensitive leverage on the MW potential, much as polar ring galaxies have been exploited to determine the properties of extragalactic systems (see, for example, Sparke 2002). However, since its discovery by Ibata, Gilmore, \& Irwin (1994), Sgr has been difficult to explore because it is expansive and its core lies partly obscured by foreground dust and stars of the Galactic disk and bulge. The Two Micron All Sky Survey (2MASS) remedies both problems by its complete sky coverage and the reduced effects of extinction in the near infrared (NIR). Moreover, Sgr contains a significant population of M-giant stars, which are not only luminous in the NIR, but separable from foreground dwarfs in the 2MASS $\left(J-H, J-K_{\mathrm{s}}\right)$ diagram. MSWO showed that Sgr is the primary contributor $(>75 \%)$ of $J-K_{\mathrm{S}}>1.0$ M-giants away from the MW disk. All-sky maps of 2MASS M giants reveal Sgr and its nearly polar (tipped by $76.5^{\circ}$ from the disk) tidal-tail system in vivid detail, wrapping $360^{\circ}$ around the sky. Figure 1 , made by adopting the M-giant colour-magnitude relation from the $\mathrm{Sgr}$ core (assumed to be at $24 \mathrm{kpc}$ ) to obtain photometric parallaxes, shows the leading arm of the Sgr system to be falling down onto the Galactic plane with an apparently near 'direct hit' on the solar neighborhood. Assuming a 47

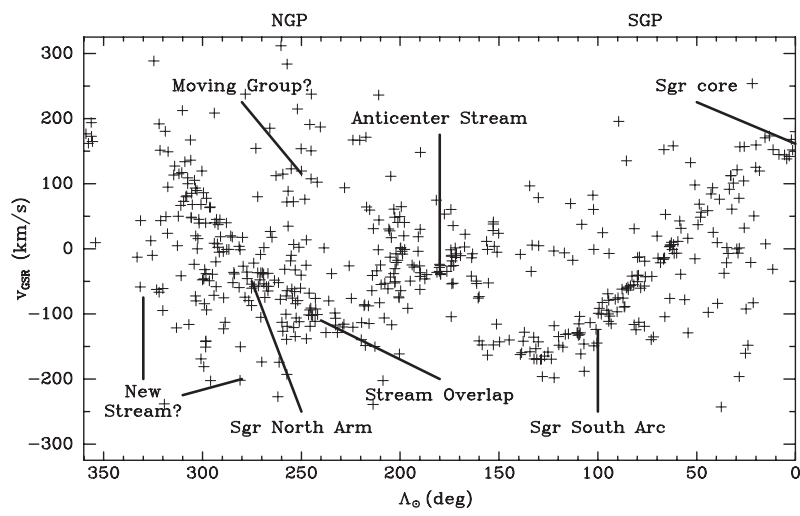

Figure 2 Radial velocities (in the Galactic Standard of Rest) of M-giants more distant than $10 \mathrm{kpc}$ (selected mainly from the Figure 1 sample) as a function of Sgr orbital longitude, $\Lambda$, defined in Figure 3. The approximate directions of the North and South Galactic Poles (NGP and SGP) are indicated at the top.

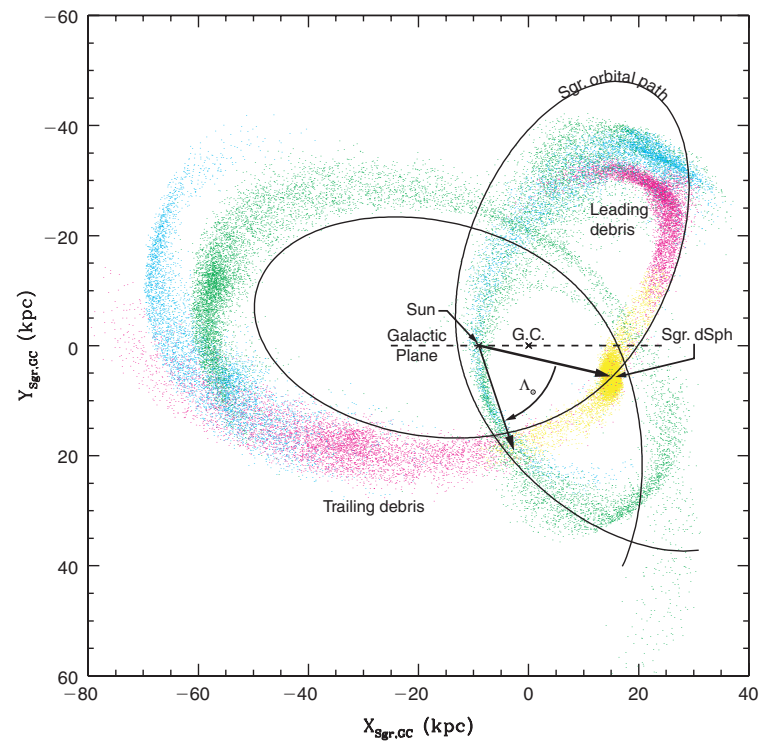

Figure $3 N$-body simulation of the disruption of the Sgr system constrained by the M-giant data. Model parameters are given in Law et al. (2003) and Majewski et al. (2003a). Different colours correspond to debris lost in successive Sgr perigalacticon passages (with yellow, magenta, cyan, green representing the most recent to oldest stripped debris, respectively). The definition of the origin of the $\Lambda_{\odot}$ longitude coordinate is shown by the arrow pointing to the Sgr dSph core. $\Lambda_{\odot}$ increases in the direction of trailing debris.

Tuc luminosity function to extrapolate the NGP M-giant density to lower luminosity stars, this suggests that there could be at least one alien Sgr star within $30 \mathrm{pc}$ of us.

Majewski et al. (2004) have been obtaining radial velocities of Figure $1 \mathrm{M}$-giants along the Sgr arms using the Swope 1-m, KPNO 2.1-m, YALO 1.5-m, and Bok $2.3-\mathrm{m}$ telescopes. Hundreds of these $\sim 5-\mathrm{km} \mathrm{s}^{-1}$ precision velocities (Figure 2 ) clearly delineate the Sgr trailing (South) and leading (North) arms in velocity space. Such data provide critical constraints on $\mathrm{Sgr} N$-body simulations developed to understand in detail the Sgr interaction with the MW (Law et al. 2004). Figure 3 shows that these models can quite closely match the observational data 
(compare to Figure 1), and indicate that the M-giants trace only the Sgr debris created in the past $\sim 2$ Gyr (yellow, magenta, and cyan points in Figure 3) of a 0.7-Gyr period Sgr orbit. This makes sense, since the Sgr age-metallicity relation (Layden \& Sarajedini 2000) implies that the Mgiants correspond to a population created in the Sgr core only a few Gyr ago. Thus, any older Sgr arms (e.g., corresponding to the green debris in Figure 3 ) will be better traced with older stellar tracers.

Law et al. (2003) and Majewski et al. (2003a, 2004) discuss preliminary work to use the extensive tidal tail system revealed by $2 \mathrm{MASS}$ M-giants as dynamical probes of the MW potential - its size, halo flattening, and lumpiness - and the global character of the Sgr dwarf — its orbit, mass, and dark matter content. Somewhat surprising results from this work are that the Galactic potential appears to be quite spherical and may not be particularly lumpy, both findings in contrast to expectations from the nominal halos produced in CDM simulations.

\section{Other M-Giant Tidal Streams}

It is interesting that the Magellanic Clouds - two huge reservoirs of M-giants - have no obvious M-giant tails themselves. However, other tidal streams reveal themselves in the spatial and dynamical 2MASS M-giant data. We note in Majewski et al. (2004) that some of the stars more widely spread in $v_{\mathrm{GSR}}$ on the right hand side of Figure 2 are consistent with expectations for wrapped, leading arm stars overlapping the trailing Sgr arm in the Southern Hemisphere. Indeed, if, as is suggested by Figure 1, the Sgr leading arm passes through the solar neighborhood, we should expect to see it as a flow of stars perpendicular to the disk. We have observed a corresponding net North to South radial-velocity dipole among a sample of hundreds of closer $(4<d<7 \mathrm{kpc}) 2 \mathrm{MASS}$ M-giants, but the dipole signal — of magnitude roughly $50 \mathrm{~km} \mathrm{~s}^{-1}$ towards the SGP - appears to be diluted from the expected $\sim 250 \mathrm{~km} \mathrm{~s}^{-1}$. This may be due to contamination from thick-disk M-giants as well as to projection effects, but possibly other, overlapping tidal-debris populations could be contributing to the dilution.

Another strong M-giant feature lying across the center of Figure 2 corresponds to stars from the 'Monoceros' (Mon) structure first identified by Newberg et al. (2002) within the Sloan Digital Sky Survey (SDSS). Early discussions have debated whether Mon is a warp or resonance of the disk (Ibata et al. 2003), a dynamically young tidal stream (Yanny et al. 2003, hereafter Y03; Rocha-Pinto et al. 2003, hereafter R03; Crane et al. 2003, hereafter C03), or perhaps a shell of multiply wrapped, old tidal debris (Helmi et al. 2003). Once again the NIR photometry of 2MASS is helpful to understanding this heavily obscured structure, which is aligned very closely to the Galactic plane, just outside the MW disk. As shown by MSWO, Mon is prominently represented in the 2MASS M-giant maps, a fact that was exploited by R03 to show that it is a coherent structure arcing at least $180^{\circ}$ around

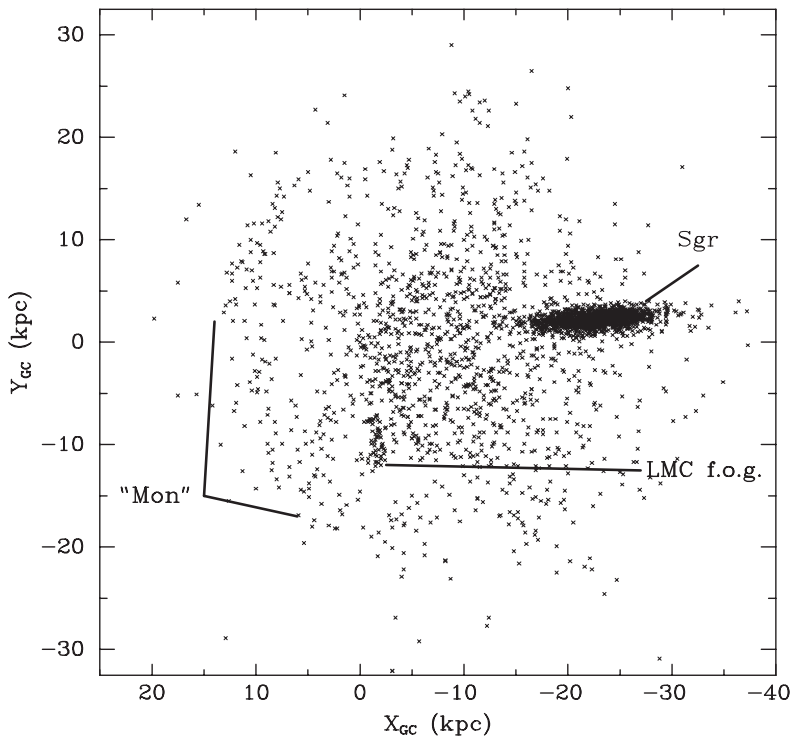

Figure 4 Galactic $X_{\mathrm{GC}}-Y_{\mathrm{GC}}$ distribution (in a sun-centred, lefthanded convention) for $J-K_{\mathrm{S}}>1.0 \mathrm{M}$ giants lying in the range $-7<Z_{\mathrm{GC}}<-4.5 \mathrm{kpc}$. The 'Mon' tidal structure is visible as the arc of stars seen prominently in the second and third Galactic quadrants and beyond. The Sgr core (to the right) is also intercepted by this slice of the Galaxy. The feature marked 'LMC f.o.g.' is a 'finger of God' artifact that is the result of using a Sgr colour-absolute magnitude relation for all M-giants, which is inappropriate to M-giants in the Magellanic Clouds.

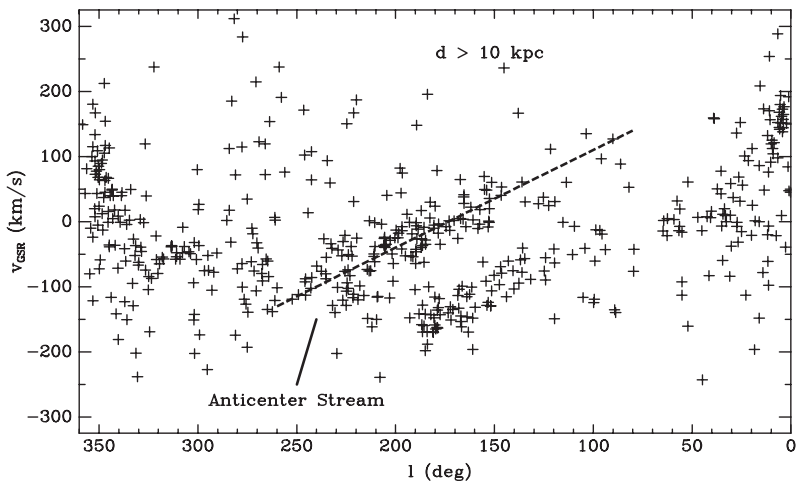

Figure 5 Radial velocities (in the Galactic Standard of Rest) of M-giants more distant than $10 \mathrm{kpc}$ (selected mainly from the Figure 1 sample) as a function of Galactic longitude. Stars corresponding to the 'Mon' ring fall roughly along the solid line. The linear grouping of stars below this are from the Sgr trailing arm.

the outer periphery of the MW disk in the second and third Galactic quadrants (see also Figure 4). C03 supplemented this analysis with spectroscopy of Mon M-giants (included in Figures 2 and 5), and found a radial-velocity trend with Galactic longitude similar to expectations for a Sgr-like stream in a low inclination, slightly non-circular orbit. The true velocity coherence of this feature is belied by the projection along the $S g r$ plane in Figure 2, a plane almost perpendicular to the Mon orbital plane. When the Figure 2 data are cast in a projection more appropriate to Mon (Figure 5), the velocity coherence of the structure is more obvious (note the corresponding loss of coherence for Sgr debris in this figure) and the velocity trend is then 
seen to have more of the clearly defined, 'Sgr-like' arc with position on the sky (albeit with about double the velocity dispersion of the Sgr stream).

The Mon M-giant spectra reveal stars more metal poor than the Sgr M-giants $([\mathrm{Fe} / \mathrm{H}] \sim-0.4$ for Mon versus $\sim-0.2$ dex for Sgr), but, like Sgr, Mon appears to have stars spanning a large metallicity range, given the discovery of $[\mathrm{Fe} / \mathrm{H}]=-1.6$ Mon stars by Y03. Mon, like Sgr, appears to have at least four globular clusters (C03). However, unlike Sgr, Mon also seems to contain younger star clusters that have traditionally been categorized as either MW 'old open clusters' or 'transitional clusters' between the open and globular classes (Frinchaboy et al. 2004). The weight of evidence seems to favour Mon as a tidally disrupting dwarf galaxy with extensive debris arms like the Sgr system, as previously discussed by Y03. Unfortunately, even with 2MASS this structure is difficult to study, although work by R03 and Rocha-Pinto et al. (in preparation) shows that the true centre of this system is actually many dozens of degrees away from the constellation of Monoceros.

Figure 2 suggests the presence of other radial velocity structures, particularly in the Northern Hemisphere (one potential 'stream' having a more negative $v_{\mathrm{GSR}}$ than Sgr is indicated in Figure 2, but these stars are also consistent with being Sgr leading arm stars passing inside the solar circle). That these other streams seem less coherent than the Sgr stream in Figure 2 could be a reflection of the same projection problem demonstrated above (and in Figure 5) with the Mon stream. As we continue to collect more Mgiant velocities, we hope to better define these possible new halo substructures. However, the Figure 2 suggestion of a few possible streams towards the NGP is reminiscent of the similar finding of three apparent halo streams in the magnitude-limited phase space analysis of the nearby halo towards the NGP by Majewski, Munn, \& Hawley (1996).

\section{K-Giant Substructure in the Distant Halo}

Figures 2 and 5 show examples of the velocity-angle trends exhibited by coherent tidal streams and what a halo networked by only a few such streams looks like in velocity-position space. However, because M-giants are a relatively rare population in MW satellites (only relatively metal-enriched populations produce M-giants), it is likely that many (most) MW streams are not represented by the Galactic cross-sections presented in those M-giant views. A systematic search of the halo using $K$-giant tracers, however, should reveal all existing, luminous substructure. Using the Washington $M, T_{2}+D D O 51$ technique we (for example, Majewski 2003; Majewski et al., in preparation) have undertaken a pilot survey for tidal streams in a set of 33 fields that cross-section the Galactic halo along a $20-25^{\circ}$ radius cone extending from the Sun and encircling the Magellanic Clouds. While one goal of this work is to search for stellar tidal debris from the Clouds, by doing a magnitude-limited photometric and spectroscopic survey of all giants in these fields we create a dynamical cross-section of all

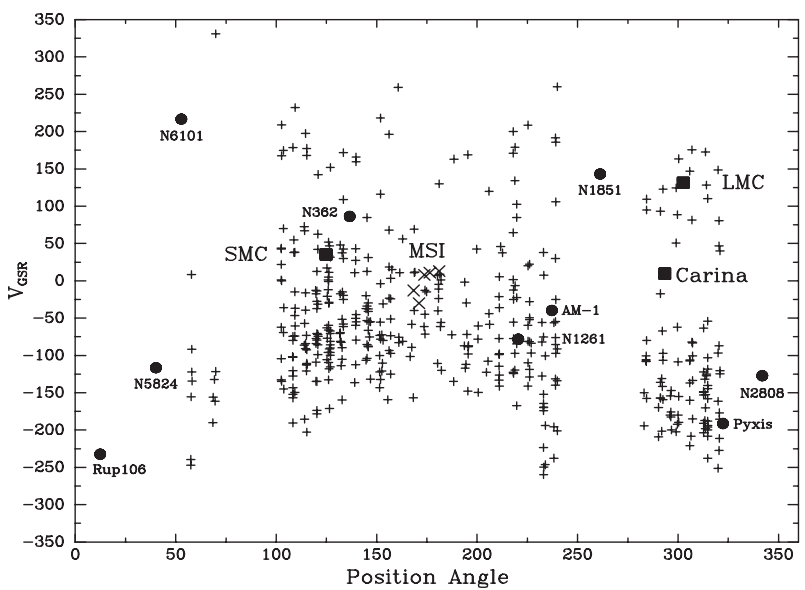

Figure 6 Galactic Standard of Rest radial velocities of K-giants in a magnitude-limited survey of fields positioned along a roughly circular ring of radius $\sim 20-25^{\circ}$ centered on a point directly between the two Magellanic Clouds (i.e., all survey fields are outside of the positions of both Clouds). The position angle coordinate refers to the angular position of the stars around this circle, with $P A=0$ due North in celestial coordinates. Various star clusters in this part of the sky (circles), as well the LMC, SMC, Carina (all shown as squares) and points within Magellanic Stream Cloud I (crosses), are indicated.

substructure in this direction of the Galaxy. Photometric catalogues covering $0.5-1.0 \mathrm{deg}^{2}$ per field were created with the Swope telescope, and spectra of identified giant stars to $M \sim 18.5$ in each field have been obtained with the Du Pont 2.5-m telescope. These data reveal a halo rich in substructure, with numerous fat and narrow arc-like, velocity-position correlations, as expected for an outer halo dominated by networked tidal streams (Figure 6). A striking aspect of the velocity distributions in Figure 6 is that many fields show no sign of a random, dynamically hot field population, which should have a concentration of stars centred at a mean Galactocentric velocity of zero. This strongly suggests (1) that the outer halo of $\mathrm{K}$-giants has derived almost completely from accretion, and (2) that tidal tails remain coherent for a long time in the outer halo, so that it remains not well-mixed today.

The structures observed in Figure 6 have a variety of velocity dispersions, distances (from 10 to $100 \mathrm{kpc}$ ), lengths (from 'moving groups' that intersect only one survey field to structures coherently spanning fields over dozens of degrees), and apparent metallicities (including some structures with stars having extremely metal-poor spectra). Some features coincide with expectations for pieces of the Magellanic Clouds; for example a relatively 'hot' group of giant stars (with dispersion about $50 \mathrm{~km} \mathrm{~s}^{-1}$ ) clustered around the LMC velocity and position. Additional Washington +DDO51 photometric crosssectioning to the northeast of the LMC clearly shows what looks to be either a very extended halo of LMC giant stars out to $20^{\circ}$ or LMC/SMC tidal debris there. The SMC itself appears to share the radial velocity of another of the velocity-position arcs. Whether these specific substructures represent actual Magellanic tidal debris remains to be determined through modelling and additional 
observations of giants nearer the Clouds. We also find a small number of halo giants with velocities and positions coincident with the H I Magellanic Stream, perhaps supporting the notion that it is tidal in origin (e.g., Putman et al. 1998; although cf. Maddison, Kawata, \& Gibson 2002); however, the density of such stars is low. One drawback of the limited area coverage of the current survey is that the apparent tidal structures cannot be followed for more than a few dozen degrees and, due to the circular placement of the fields, the true orientation of structures 'glancing' the ring of fields cannot yet be ascertained.

We are remedying these shortcomings through the Grid Giant Star Survey (GGSS), a partially filled, evenly sampled, 'all-sky', $M, T_{2}+D D O 51$ survey of the halo reaching $V=17.5$ or more. Imaging of the 1302 fields over $0.5-1.0 \mathrm{deg}^{2}$ per field is now virtually complete and reduced to catalogues. Spectroscopic follow-up has begun with the Hydra spectrographs on NOAO telescopes. A pilot sampling of bright giants in GGSS fields by Kundu et al. (2002) has already found new halo tidal debris relatively near the Sun that may be an older, wrapped tail from the Sgr dwarf.

\section{Concluding Remarks}

The Sgr core and tail system is a Rosetta Stone of the process of accretion-driven halo building, but is clearly just one, now well-studied fixture of what is a common element of the MW halo. Our observational picture of halo formation should reach dramatic levels of detail in the near future, not only from the wealth of tidal debris already uncovered, but from further riches derived from mining large surveys like SDSS, 2MASS, RAVE, AMEX, SIM, Gaia, and the GGSS. Pervasive halo substructure in the MW (and M31) points to the commonality of minor mergers in the formation of large disk galaxies. The luminous victims of these mergers probably resemble the present MW satellites, whose 'King+break' profiles mimic the structure of the disrupting Sgr core, a fact that suggests that today's satellites are also crumbling under the stress of Galactic tides.

\section{Acknowledgments}

I appreciate funding from NASA, the National Science Foundation, and the David and Lucile Packard Foundation. I am grateful to numerous collaborators who made possible the results presented, especially William Kunkel, who played a critical role in securing most of the velocity data; Mike Skrutskie, who lent invaluable assistance with the 2MASS projects; David Law and Kathryn Johnston, my modelling collaborators; Jamie Ostheimer, who made a number of key contributions to the various projects; Richard Patterson for numerous levels of assistance; and a multitude of other students and post-docs who helped with observing, reductions, and leading the dSph mapping projects. I am grateful to all of the coauthors of papers 'in preparation' discussed here for the privilege of presenting this work.

\section{References}

Bessell, M. S., \& Brett, J. M. 1988, PASP, 100, 1134

Crane, J. D., Majewski, S. R., Rocha-Pinto, H. J., Frinchaboy, P. M., Skrutskie, M. F., \& Law, D. R. 2003, ApJ, 594, L119

Eggen, O. J., Lynden-Bell, D., \& Sandage, A. R. 1962, ApJ, 136, 748

Frinchaboy, P. M., Majewski, S. R., Crane, J. D., Reid, I. N., Rocha-Pinto, H. J., Phelps, R. L., Patterson, R. J., \& Muñoz, R. R. 2004, ApJ, 602, L21

Helmi, A., Navarro, J. F., Meza, A., Steinmetz, M., \& Eke, V. R. 2003, ApJ, 592, L25

Ibata, R. A., Gilmore, G., \& Irwin, M. J. 1994, Natur, 370, 194

Ibata, R. A., Irwin, M. J., Lewis, G. F., Ferguson, A. M. N., \& Tanvir, N. 2003, MNRAS, 340, L21

Irwin, M., \& Hatzidimitriou, D. 1995, MNRAS, 277, 1354

Johnston, K. V., Choi, P. I., \& Guhathakurta, P. 2002, AJ, 124, 310

Johnston, K. V., Sigurdsson, S., \& Hernquist, L. 1999, MNRAS, 302,771

Kauffmann, G., White, S. D. M., \& Guiderdoni, B. 1993, MNRAS, 264, 201

King, I. R. 1962, AJ, 67, 471

Klypin, A., Kravtsov, A. V., Valenzuela, O., \& Prada, F. 1999, ApJ, 522,82

Kuhn, J. R., Smith, H. A., \& Hawley, S. L. 1996, ApJ, 469, L93

Kundu, A., et al. 2002, ApJ, 576, L125

Law, D. R., Johnston, K. V., \& Majewski, S. R. 2004, ApJ, submitted

Law, D. R., Majewski, S. R., Skrutskie, M. F., \& Johnston, K. V. 2003, in ASP Conf. Ser., Tidal Tails and Galactic Satellites, eds. D. Martinez-Delgado, \& F. Prada (San Francisco: ASP), in press (astro-ph/0309567)

Layden, A., \& Sarajedini, A. 2000, AJ, 119, 1760

Maddison, S. T., Kawata, D., \& Gibson, B. K. 2002, Ap\&SS, 281,421

Majewski, S. R. 2003, in ASP Conf. Ser. 296, New Horizons in Globular Cluster Astronomy, eds. G. Piotto, G. Meylan, S. G. Djorgovski, \& M. Riello (San Francisco: ASP), 447

Majewski, S. R. 2004, in ASP Conf. Ser., Milky Way Surveys: The Structure and Evolution of our Galaxy, eds. D. Clemens, T. Brainerd, \& R. Shah (San Francisco: ASP), in press

Majewski, S. R., Munn, J. A., \& Hawley, S. L. 1996, ApJ, 459, L73

Majewski, S. R., Ostheimer, J. C., Kunkel, W. E., \& Patterson, R. J. 2000a, AJ, 120, 2550

Majewski, S. R., Ostheimer, J. C., Patterson, R. J., Kunkel, W. E., Johnston, K. V., \& Geisler, D. 2000b, AJ, 119, 760

Majewski, S. R., et al. 2004, AJ, in press

Majewski, S. R., Law, D. R., Johnston, K. V., Skrutskie, M. F., \& Weinberg, M. D. 2003a, in ASP Conf. Ser., IAU Symp. 220: Dark Matter in Galaxies, eds. S. Ryder, D. J. Pisano, M. Walker, \& K. Freeman (San Francisco: ASP), in press

Majewski, S. R., Skrutskie, M. F., Weinberg, M. D., \& Ostheimer, J. C. 2003b, ApJ, 599, 1082 (MSWO)

Mayer, L., Moore, B., Quinn, T., Governato, F., \& Stadel, J. 2002, MNRAS, 336, 119

Moore, B., Ghigna, S., Governato, F., Lake, G., Quinn, T., Stadel, J., \& Tozzi, P. 1999, ApJ, 524, L19

Morrison, H. L., Olszewski, E. W., Mateo, M., Norris, J. E., Harding, P., Dohm-Palmer, R. C., \& Freeman, K. C. 2001, AJ, 121,283

Navarro, J. F., Frenk, C. S., \& White, S. D. M. 1997, ApJ, 490, 493

Newberg, H. J., et al. 2002, ApJ, 569, 245

Putman, M. E., et al. 1998, Natur, 394, 752

Rocha-Pinto, H. J., Majewski, S. R., Skrutskie, M. F., \& Crane, J. D. 2003, ApJ, 594, L115

Searle, L., \& Zinn, R. 1978, ApJ, 225, 357

Sparke, L. S. 2002, in The Shapes of Galaxies and Their Dark Halos, ed. P. Natarajan (Singapore: World Scientific), 178

Walcher, C. J., Fried, J. W., Burkert, A., \& Klessen, R. S. 2003, A\&A, 406, 847

Yanny, B., et al. 2003, ApJ, 588, 824 